\title{
Insegurança Alimentar, consumo de alimentos e estado nutricional de mulheres de Campo Grande, Mato Grosso do Sul
}

\author{
Food insecurity, food consumption and nutritional status of women from \\ Campo Grande, Mato Grosso do Sul \\ Inseguridad Alimenticia, consumo de alimentos y estado nutricional de las
mujeres de Campo Grande, Mato Grosso do Sul
}

Lídia Viegas Tenório da Silva Santos ${ }^{1}$

Larissa de Azevedo Cáceres ${ }^{1}$

Giovana Eliza Pegolo ${ }^{1}$

DOI: http://dx.doi.org/10.20435/inter.v0i0.1814

\begin{abstract}
Resumo: Objetivou-se avaliar a Insegurança Alimentar de famílias, tendo a figura materna como representante, e fatores associados à saúde. Estudo transversal, com 61 mulheres de Campo Grande, MS. Constataramse $52,4 \%$ de Insegurança Alimentar, consumo de alimentos inadequado e 50,8\% de excesso de peso. A inadequação do consumo de alimentos pode favorecer a instalação de doenças crônicas e agravar o estado nutricional já evidenciado pelo expressivo percentual de excesso de peso.
\end{abstract}

Palavras-chave: consumo de alimentos; mães; obesidade; Segurança Alimentar e Nutricional.

Abstract: The objective was to evaluate the Food Insecurity of families, with the maternal figure as representative, and associated factors associated with health. This is a cross-sectional study with 61 women from Campo Grande, MS. 52.4\% of Food Insecurity, inadequate food consumption and 50.8\% of excess weight were found. The inadequacy of food consumption may favor the establishment of chronic diseases and aggravate the nutritional status already evidenced by the expressive percentage of excess weight.

Key words: food consumption; mothers; obesity; Food and Nutrition Security.

Resumen: Se objetivó evaluar la Inseguridad Alimentaria de familias, teniendo la figura materna como representante, y factores asociados a la salud. Se trata de un estudio transversal con 61 mujeres de Campo Grande, MS. Se constató un 52,4\% de Inseguridad Alimentaria, consumo de alimentos inadecuado y 50,8\% de exceso de peso. La inadecuación del consumo de alimentos puede favorecer la instalación de enfermedades crónicas y agravar el estado nutricional ya evidenciado por el expresivo porcentaje de exceso de peso.

Palabras clave: consumo de alimentos; madres; obesidad; Seguridad Alimentaria y Nutricional.

\section{INTRODUÇÃO}

A Segurança Alimentar e Nutricional no Brasil é definida como a garantia do direito de todos ao acesso regular e permanente a alimentos de qualidade, em quantidade suficiente, contudo sem comprometer o acesso a outras necessidades essenciais e tendo como base práticas alimentares promotoras de saúde, com integral respeito à diversidade cultural, ambiental, econômica e socialmente sustentáveis (BRASIL, 2006). Dentre as dimensões que integram a análise da Segurança Alimentar e Nutricional, o acesso à alimentação adequada e saudável constitui um dos aspectos que possibilitam a identificação dos grupos populacionais mais vulneráveis à violação do Direito Humano à Alimentação Adequada (INSTITUTO BRASILEIRO DE GEOGRAFIA E ESTATÍsTICA, 2014a).

Para a identificação da situação de Insegurança Alimentar, um dos instrumentos utilizados é a Escala Brasileira de Insegurança Alimentar (EBIA), a qual, além de explorar a percepção das

\footnotetext{
${ }^{1}$ Universidade Federal de Mato Grosso do Sul (UFMS), Campo Grande, Mato Grosso do Sul, Brasil
} 
famílias em relação ao acesso aos alimentos, mostra-se efetiva para estimar a prevalência dos diversos níveis de Insegurança Alimentar (IA), permitindo a identificação de grupos em risco e de determinantes e consequências. Além disso, a investigação de aspectos físico-biológicos, como o estado nutricional, constitui instrumento importante para estudos exploratórios dos fatores coadjuvantes da Segurança Alimentar e Nutricional (KEPPLE; SEGALL-CORRÊA, 2011). Tal fato, de acordo com o II Plano Nacional de Segurança Alimentar e Nutricional 2016-2019 (CÂMARA INTERMINISTERIAL DE SEGURANÇA ALIMENTAR E NUTRICIONAL [CAISAN], 2016), fundamenta-se, entre outras preocupações, na necessidade de controlar e prevenir agravos à saúde decorrentes da alimentação inadequada, como o excesso de peso, amplamente reconhecido como fator de risco para Doenças Crônicas Não Transmissíveis (DCNTs).

Sobre o estado nutricional e suas consequências à saúde, diversos fatores podem contribuir para sua adequação ou não, como a falta de acesso a alimentos saudáveis, as escolhas alimentares, a não diversificação dos alimentos consumidos e a maior oferta de alimentos não saudáveis e/ou o acesso facilitado a esses alimentos, os quais impactam no desenvolvimento de doenças ao longo dos anos (MAZUR; NAVARRO, 2015).

No que diz respeito à alimentação, o consumo de alimentos de forma inadequada, tanto qualitativamente quanto quantitativamente, apresenta impacto negativo para a saúde desde a primeira década de vida. No caso das frutas e verduras, de acordo com prospecções da Organização Mundial da Saúde (OMS), estima-se que o baixo consumo cause cerca de 2,7 milhões de mortes a cada ano, constituindo um dos dez maiores fatores de risco que contribuem para a mortalidade por DCNTs como diabetes mellitus tipo 2, dislipidemias, hipertensão arterial sistêmica e a obesidade (WORLD HEALTH ORGANIZATION, 2002).

Tendo em vista que as mulheres assumem responsabilidades na sociedade e nos núcleos familiares, especialmente com relação ao consumo de alimentos e à saúde da família, objetivou-se avaliar a Insegurança Alimentar, o estado nutricional e os fatores associados à referida condição em um grupo de mulheres, representado por mães de crianças matriculadas em um Centro de Educação Infantil de Campo Grande, MS.

\section{METODOLOGIA}

Trata-se de estudo transversal, constituído por amostra de conveniência de mães de crianças matriculadas em um Centro de Educação Infantil da rede municipal de ensino de Campo Grande, MS. Todas as mulheres com idade igual ou superior a 18 anos foram convidadas a participarem do estudo. Os dados foram coletados no período de maio a julho de 2015, por meio de entrevistas individuais, realizadas apenas com as mães, sendo a figura materna considerada, nesta investigação, como representante da família e principal responsável pelos cuidados em relação à alimentação da família.

Para a investigação da Insegurança Alimentar, foi aplicada a EBIA, validada para a população brasileira por Segall-Corrêa et al. (2004) e adotada também pela Pesquisa Nacional por Amostra de Domicílios (PNAD) (INSTITUTO BRASILEIRO DE GEOGRAFIA E ESTATÍSTICA, 2014a). Foram considerados os seguintes pontos de corte para famílias com menores de 18 anos: Segurança Alimentar - 0 pontos; IA Leve - 1 a 5 pontos; IA Moderada - 6 a 9 pontos e IA Grave - 10 a 14 pontos.

A avaliação do consumo de alimentos foi realizada por meio de questões previamente selecionadas, adaptadas da pesquisa Vigilância de Fatores de Risco e Proteção para Doenças Crônicas 
por Inquérito Telefônico (VIGITEL), considerando consumo de alimentos de risco (consumo de refrigerantes ou sucos artificiais, doces e carne vermelha com gordura) ou proteção (consumo de feijão, frutas, verduras e leite) para DCNTs (BRASIL, 2015). Além disso, as participantes foram questionadas sobre a responsabilidade pelo consumo de alimentos da família. Esse questionamento fundamentou-se no estreito vínculo entre a figura materna e os demais membros da família no que diz respeito à alimentação e perpetuação de hábitos alimentares.

A renda familiar foi estimada por meio da aplicação do Critério de Classificação Econômica Brasil, desenvolvido pela Associação Brasileira de Empresas de Pesquisa (2014). Esse instrumento avalia a quantidade de itens de conforto (automóvel, geladeira, entre outros) e o grau de instrução do chefe da família para identificar a classificação econômica da família. Para identificar o chefe da família, as participantes deveriam considerar o membro da família que para elas apresentasse essa representatividade. Foram considerados os seguintes pontos de corte e respectivos valores (em reais), associados à Renda Média Bruta Familiar Mensal, conforme valores estimados para o ano no qual o questionário foi desenvolvido: Classes A1 e A2 - 35 a 46 pontos (R\$11.037,00); Classe B1 - 29 a 34 pontos (R\$ 6.006,00); Classe B2 - 23 a 28 pontos ( $R \$ 3.118,00$ ); Classe C1 - 18 a 22 pontos (R\$ 1.865,00); Classe C2 - 14 a 17 pontos (R\$ 1.277,00); Classes D/E - 0 a 13 pontos ( $R \$ 895,00)$; em ordem decrescente economicamente.

A avaliação do estado nutricional foi realizada por meio do Índice de Massa Corporal (IMC) e Circunferência da cintura. O peso foi aferido por meio de balança digital e portátil, com capacidade de $150 \mathrm{Kg}$ e escala de $100 \mathrm{~g}$. A aferição da altura foi realizada por meio da utilização de estadiômetro portátil, com capacidade para $210 \mathrm{~cm}$, equipamento específico apenas para a mensuração da altura. A Circunferência da cintura foi aferida com fita métrica inelástica com capacidade de $150 \mathrm{~cm}$. As medidas foram realizadas em duplicata. Os procedimentos para aferição das referidas medidas e a classificação dos valores obtidos seguiram as recomendações da Organização Mundial da Saúde (WORLD HEALTH ORGANIZATION, 1995; 2008).

As variáveis foram apresentadas em frequência simples e realizou-se a associação entre os fatores analisados por meio do Teste de Qui-quadrado, a partir da utilização do programa BioEstat 5.3. Adotou-se o nível de significância de 5\% para assumir as associações como estatisticamente significantes $(p<0,05)$.

Este estudo foi aprovado pelo Comitê de Ética em Pesquisa em Seres Humanos da Universidade Federal de Mato Grosso do Sul (UFMS). Após esclarecimentos a respeito dos objetivos e de todos os procedimentos do estudo, as mães foram convidadas a participarem voluntariamente e passaram a integrar a amostra aquelas que manifestaram aceitação por meio da assinatura do Termo de Consentimento Livre e Esclarecido.

\section{RESULTADOS}

Participaram integralmente deste estudo 61 mães (sendo que do total de possíveis participantes, $n=146,30 \%$ desistiram, $11 \%$ recusaram e $17 \%$ estiveram ausentes durante todo 0 período da coleta de dados). A seguir são apresentados os resultados descritivos das participan-

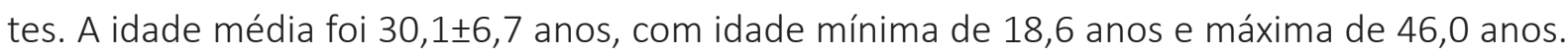
O número médio de filhos com idade inferior a 18 anos foi 2,0 $0 \pm 0,9$ anos. Para os dados antropométricos, foram observados os seguintes valores médios e desvio padrão: peso $=66 \pm 14,3 \mathrm{~kg}$; altura $=1,60 \pm 0,05$ metros; $I M C=25,8 \pm 5,6 \mathrm{~kg} / \mathrm{m}^{2}$ e Circunferência da cintura $=83,4 \pm 10,9 \mathrm{~cm}$. 
Segundo a classificação econômica adotada, 47,5\% (n=29) da amostra encontrava-se na classe C1 e 22,9\% ( $n=14)$ na C2, com estimativa de Renda Média Bruta Familiar Mensal de R\$ 1.865,00 e $\mathrm{R} \$ 1.277,00$, respectivamente. As demais participantes foram classificadas da seguinte maneira: $16,4 \%(n=10)$ na classe $B 2,6,6 \%(n=4)$ na $B 1$ e $6,6 \%(n=4)$ na classe $D$, com rendas de $R \$ 3.118,00$, $\mathrm{R} \$$ 6.006,00 e R\$ 895,00, em sequência. Nenhuma participante foi identificada nas classes A e E.

Com relação à escolaridade, $32,7 \%(n=20)$ das mulheres entrevistadas afirmaram ter Ensino Médio Completo e 21,3\% ( $n=13)$ Ensino Médio Incompleto. Apenas 11,5\% ( $n=7)$ registraram Ensino Superior Completo. Quanto aos demais anos de escolaridade, Ensino Fundamental Incompleto e Completo e Ensino Superior Incompleto, para cada um dos níveis constataram-se $11,5 \%$ ( $n=7$, para cada um).

Não foram constatadas diferenças significativas entre classificação econômica e estado nutricional e entre escolaridade e estado nutricional da mãe $(p>0,05)$.

Quanto à escolaridade do chefe da família, 47,5\% (n=29) possuíam Ensino Fundamental Completo; 34,4\% ( $n=21)$, Ensino Médio Completo; 11,5\% ( $n=7)$, Ensino Superior Completo; e $6,6 \%(n=4)$, Ensino Fundamental Incompleto.

Os resultados obtidos por meio da EBIA são apresentados na Tabela 1. Destaca-se a proporção de famílias, 52,4\% (n=32), com algum grau de IA (Insegurança Alimentar Leve, Moderada e Grave).

Tabela 1 - Distribuição da classificação da Segurança Alimentar e da severidade da Insegurança Alimentar de acordo com a Escala Brasileira de Insegurança Alimentar (EBIA) de mulheres ( $n=$ 61) de Campo Grande, MS, 2015

\begin{tabular}{l|c}
\hline \multicolumn{1}{c}{ Classificação - Segurança/Insegurança Alimentar } & Distribuição das mulheres \\
\cline { 2 - 2 } & $\mathbf{n}(\%)$ \\
\hline Segurança Alimentar & $29(47,5)$ \\
Insegurança Alimentar Leve & $25(41,0)$ \\
Insegurança Alimentar Moderada & $6(9,8)$ \\
Insegurança Alimentar Grave & $1(1,6)$ \\
\hline Total & $61(100,0)$ \\
\hline
\end{tabular}

$\mathrm{n}=$ número de participantes de acordo com a classificação em Segurança ou Insegurança Alimentar.

Fonte: Elaborado pelas autoras.

Cabe ressaltar que não foi constatada diferença significativa entre IA e estado nutricional $(p=0,4637)$ e entre IA e escolaridade da mulher $(p=0,5410)$.

Ao investigar a associação entre a escolaridade do chefe da família e a Segurança Alimentar, o número de chefes de família com Ensino Fundamental Completo e em condição de Segurança Alimentar foi significativamente maior em relação às demais classificações de escolaridade $(p=0,0174)$. Cabe esclarecer que a participante, de acordo com sua referência pessoal para chefe da família, o que não necessariamente significa que seja o pai/marido, indicou a escolaridade, item este contemplado no Critério de Classificação Econômica Brasil, adotado para a estimativa da renda familiar.

A análise das questões da EBIA individualmente (Tabela 2) possibilitou destacar os seguintes resultados: $36,1 \%$ ( $n=22$ ) das famílias apresentaram preocupação que o alimento acabasse antes que tivessem dinheiro para comprar mais (questão 1); 18\% ( $n=18)$ afirmaram que o alimento acabou antes de obterem recursos financeiros para nova aquisição (questão 2); 36,1\% (n=22) 
destacaram que ficaram sem condições financeiras para terem uma alimentação saudável e adequada nos três meses anteriores ao estudo (questão 3); e 37,7\% ( $n=23)$ dos moradores tiveram que fazer ajustes quantitativos na alimentação uma vez que o dinheiro findou (questão 4). As questões 9 e 10, que abordam a condição de Segurança Alimentar em menores de 18 anos, especificamente quanto à alimentação saudável e variada e a disponibilidade de apenas alguns poucos tipos de alimentos por falta de dinheiro, também apresentaram destaque nos resultados afirmativos para IA, ambas com $16,4 \%(n=10)$ de respostas afirmativas.

Tabela 2 - Distribuição das respostas das mulheres entrevistadas com base na Escala Brasileira de Insegurança Alimentar (EBIA), Campo Grande, MS, 2015

\section{Escala Brasileira de Insegurança Alimentar}

$\operatorname{Sim}$ Não

1. Nos últimos três meses, os moradores deste domicílio tiveram a preocupação de que a comida acabasse antes que tivessem dinheiro para $\quad 22(36,1) \quad 39(63,9)$ comprar mais comida?

2. Nos últimos três meses, os alimentos acabaram antes que os moradores desse domicílio tivessem dinheiro para comprar mais comida?

$11(18,0) \quad 50(82,0)$

3. Nos últimos três meses, os moradores desse domicílio ficaram sem dinheiro para ter uma alimentação saudável e variada?

$22(36,1) \quad 39(63,9)$

4. Nos últimos três meses, os moradores deste domicílio comeram apenas alguns poucos tipos de alimentos que ainda tinham, porque o dinheiro acabou?

5. Nos últimos três meses, algum morador de 18 anos ou mais de idade deixou de fazer alguma refeição porque não havia dinheiro para comprar a comida?

6. Nos últimos três meses, algum morador de 18 anos ou mais de idade comeu menos do que achou que devia, porque não havia dinheiro para comprar comida?

$3(4,9) \quad 58(95,1)$

7. Nos últimos três meses, algum morador de 18 anos ou mais de idade sentiu fome, mas não comeu, porque não tinha dinheiro para comprar comida?

$2(3,3) \quad 59(96,7)$

8. Nos últimos três meses, algum morador de 18 anos ou mais de idade ficou um dia inteiro sem comer ou, teve apenas uma refeição ao dia, porque não tinha dinheiro para comprar a comida?

$2(3,3) \quad 59(96,7)$

9. Nos últimos três meses, os moradores com menos de 18 anos de idade não puderam ter uma alimentação saudável e variada, porque não havia dinheiro para comprar comida?

10. Nos últimos três meses, os moradores menores de 18 anos de idade comeram apenas alguns poucos tipos de alimentos que ainda havia neste domicílio, porque o dinheiro acabou?

11. Nos últimos três meses, algum morador com menos de 18 anos de idade comeu menos do que você achou que devia, porque não havia dinheiro para comprar a comida?

12. Nos últimos três meses, foi diminuída a quantidade de alimentos das refeições de algum morador com menos de 18 anos de idade, porque não havia dinheiro suficiente para comprar a comida?

13. Nos últimos três meses, algum morador com menos de 18 anos de idade deixou de fazer alguma refeição, porque não havia dinheiro para comprar a comida?

14. Nos últimos três meses, algum morador com menos de 18 anos de idade sentiu fome, mas não comeu porque não havia dinheiro para comprar mais comida? 
Os resultados indicativos do consumo de alimentos (Tabela 3) são apresentados de acordo com a frequência semanal de consumo (número de dias na semana). Entre os alimentos considerados protetores para DCNTs, destaca-se que apenas $8,2 \%(n=5), 13,1 \%(n=8)$ e $14,8 \%$ $(n=9)$ relataram consumo diário de frutas, salada crua e leite, respectivamente; e 50,8\% ( $n=31)$ afirmaram consumir diariamente feijão. Por outro lado, alimentos considerados de risco para o desenvolvimento de DCNTs, especialmente diante de consumo frequente e em quantidades excessivas, mostraram-se frequentes por proporções importantes de participantes, como o consumo de refrigerantes ou sucos artificiais e de doces em geral $(41 \%, n=25$; e 27,9\%, $n=17$, mencionaram consumo de 5 a 7 dias/semana, respectivamente).

Tabela 3 - Frequência de consumo de alimentos, considerados como fatores de risco ou proteção para Doenças Crônicas Não Transmissíveis, das mulheres representantes de famílias de Campo Grande, MS, 2015

\begin{tabular}{lcccccccc}
\hline $\begin{array}{c}\text { Frequência } \\
\text { semanal de } \\
\text { consumo }\end{array}$ & $\begin{array}{c}\text { Feijão } \\
\text { (\%) }\end{array}$ & $\begin{array}{c}\text { Salada } \\
\text { Crua* } \\
\text { (\%) }\end{array}$ & $\begin{array}{c}\text { Frutas } \\
\text { (\%) }\end{array}$ & $\begin{array}{c}\text { Carne } \\
\text { Vermelha } \\
\text { (\%) }\end{array}$ & $\begin{array}{c}\text { Carne de } \\
\text { Frango } \\
\text { (\%) }\end{array}$ & $\begin{array}{c}\text { Refrigerantes } \\
\text { ou Sucos } \\
\text { Artificiais } \\
\text { (\%) }\end{array}$ & $\begin{array}{c}\text { Leite } \\
\text { (\%) }\end{array}$ & $\begin{array}{c}\text { Doces } \\
\text { (\%) }\end{array}$ \\
\hline 1 a 2 dias & 6,6 & 23,0 & 39,3 & 9,8 & 45,9 & 23,0 & 24,5 & 23,0 \\
3 a 4 dias & 16,4 & 32,8 & 29,6 & 24,6 & 39,3 & 19,7 & 16,4 & 26,2 \\
5 a 6 dias & 24,6 & 19,7 & 9,8 & 18,0 & 6,6 & 24,6 & 14,8 & 16,4 \\
Todos os dias & 50,8 & 13,1 & 8,2 & 45,9 & 4,9 & 16,4 & 14,8 & 11,5 \\
Quase nunca & 1,6 & 9,8 & 13,1 & 1,7 & 3,3 & 13,1 & 13,1 & 21,3 \\
Nunca & 0,0 & 1,6 & 0,0 & 0,0 & 0,0 & 3,2 & 16,4 & 1,6 \\
\hline
\end{tabular}

* Salada de alface e tomate ou verdura crua.

Fonte: Elaborado pelas autoras.

Com relação ao consumo dos alimentos investigados nesta pesquisa, o qual pode ser baseado na escolha e/ou na disponibilidade de renda da participante, não foi constatada diferença significativa entre a frequência de consumo de salada de alface e tomate ou outra verdura ou legume cru, consumo de refrigerante ou sucos artificiais, leite e de doces e Segurança Alimentar e os possíveis graus de IA ( $p>0,05)$.

Ao considerar a distribuição das participantes de acordo com o estado nutricional e frequência de consumo de salada de alface e tomate ou outra verdura ou legume cru, as mulheres com obesidade tendem a comer salada, com frequência diária maior, do que aquelas com outros estados nutricionais ( $p=0,0028)$.

Quanto ao consumo de carne vermelha com gordura, não houve diferença significativa quando associado ao estado nutricional, fato também observado em relação à frequência de consumo de refrigerantes ou sucos artificiais, de leite e de doces $(p>0,05)$.

Os resultados da Tabela 4 apresentam a classificação do estado nutricional de acordo com o IMC e Circunferência da cintura, com destaque para o percentual de participantes com excesso de peso $(50,8 \%)$, considerado como sobrepeso e obesidade, percentual este superior ao observado de mulheres em eutrofia (44,3\%). Para a Circunferência da cintura, somente 44,3\% das mulheres apresentaram valores adequados. Não foi constatada diferença significativa entre Segurança ou IA e estado nutricional das participantes $(p=0,4637)$. 
Tabela 4 - Estado nutricional das mulheres de acordo com o Índice de Massa Corporal (IMC) e Circunferência da cintura, Campo Grande, MS, 2015

\begin{tabular}{l|c}
\hline \multicolumn{1}{c|}{ Estado nutricional } & $\begin{array}{c}\text { Distribuição das participantes } \\
\mathbf{n}(\%)\end{array}$ \\
\hline Classificação do Índice de Massa Corporal (IMC) & $3(4,9)$ \\
Baixo peso $\left(\mathrm{IMC}<18,5 \mathrm{~kg} / \mathrm{m}^{2}\right)$ & $27(44,3)$ \\
Eutrofia $\left(\mathrm{IMC} \geq 18,5 \mathrm{e}<25,0 \mathrm{~kg} / \mathrm{m}^{2}\right)$ & $19(31,1)$ \\
Sobrepeso $\left(\mathrm{IMC} \geq 25,0 \mathrm{e}<30,0 \mathrm{~kg} / \mathrm{m}^{2}\right)$ & $12(19,7)$ \\
Obesidade $\left(\mathrm{IMC} \geq 30,0 \mathrm{~kg} / \mathrm{m}^{2}\right)$ & \\
Classificação da Circunferência da cintura & $27(44,3)$ \\
Adequado $(<80 \mathrm{~cm})$ & $16(26,2)$ \\
Risco elevado $(\geq 80 \mathrm{~cm})$ & $18(29,5)$ \\
Risco muito elevado $(\geq 88 \mathrm{~cm})$ & \\
\hline
\end{tabular}

n. total $=61$ participantes

Fonte: Elaborado pelas autoras.

No que diz respeito ao consumo de alimentos da família, 81,7\% das mulheres afirmaram assumir a responsabilidade pela alimentação de toda a família.

\section{DISCUSSÃO}

Um dos principais resultados deste estudo diz respeito à proporção de participantes em IA, uma vez que 52,4\% das famílias foram classificadas nesta condição. A PNAD, com dados de 2013, mostrou que, para Mato Grosso do Sul, 20,7\% das famílias com pelo menos um morador com idade inferior a 18 anos estavam em IA, valor abaixo da média nacional $(28,8 \%)$, considerando domicílios em áreas urbanas e rurais. Para as áreas urbanas, considerando amostra nacional, 26,1\% dos domicílios apresentavam algum grau de IA (INSTITUTO BRASILEIRO DE GEOGRAFIA E ESTATÍSTICA, 2014a). A comparação dos resultados mostra que parcela expressivamente superior das famílias da presente pesquisa vivenciam condições de vulnerabilidade nutricional.

Entre as famílias em IA, as questões da EBIA com maior percentual de respostas afirmativas (Questão 3 - refere-se à falta de dinheiro para uma alimentação saudável e variada; Questão 4 - os moradores se alimentaram com alguns poucos tipos de alimentos porque o dinheiro acabou; Questão 9 - os moradores com idade inferior a 18 anos não puderam se alimentar de forma saudável e variada pois o dinheiro acabou; Questão 10 - os moradores menores de 18 anos comeram apenas alguns poucos tipos de alimentos; sendo as duas últimas em domicílios com menores de 18 anos e considerando os últimos 3 meses para as quatro questões citadas), mostraram que a IA apresentou-se relacionada ao déficit financeiro, aspecto este que pode interferir tanto quantitativamente como qualitativamente na alimentação.

Quanto à influência da renda na condição de Segurança Alimentar, estudo realizado com famílias da Paraíba, conduzido em dois momentos (2005; 2011), agrupou os domicílios em duas categorias: Segurança Alimentar/IA Leve e IA Moderada/Grave. Os autores verificaram que o número de famílias em Segurança Alimentar e IA Leve apresentou acréscimo significativo no último ano estudado, em detrimento da redução dos casos de IA Moderada e Grave, decorrente do aumento na renda mensal, possibilitando às famílias a saída da condição de pobreza (CABRAL et al., 2014). 
A renda é considerada uma das variáveis com maior associação com a Segurança Alimentar, sendo tão importante que, conforme análises conduzidas com o banco de dados da PNAD de 2004, a cada 10 reais acrescidos à renda familiar mensal, aumentava-se a chance de Segurança Alimentar no domicílio em 8\% (SEGALL-CORRÊA et al., 2008).

Embora, no presente estudo, 47,5\% das famílias tenham sido classificadas em Segurança Alimentar, não podemos afirmar que estejam isentas de vulnerabilidade alimentar e nutricional. Tal observação fundamenta-se nos resultados constatados em relação ao consumo de alimentos, notadamente pelo reduzido consumo de saladas, frutas e leite e presença constante de refrigerantes e doces por proporções consideráveis das entrevistadas. Esses aspectos conduzem também ao questionamento da compreensão das mulheres a respeito do que constitui uma alimentação saudável.

Outro fato diz respeito à dificuldade de plena compreensão das questões da EBIA, pois as participantes manifestaram questionamentos que denotavam não entendimento, mesmo considerando que, no presente estudo, a aplicação da escala foi realizada pelas pesquisadoras. Cabe registrar que Voci e Slater (2015), com famílias de Piracicaba, obtiveram preenchimento incompleto da EBIA, autopreenchida pela pessoa de referência do domicílio. As autoras levantaram a hipótese de esse fato ser causado pelo difícil entendimento das perguntas da escala, possivelmente decorrente do nível de escolaridade do entrevistado.

Outro aspecto central diretamente relacionado ao conceito de Segurança Alimentar e Nutricional, além do acesso aos alimentos, é a qualidade dos alimentos que irão integrar a alimentação rotineira dos indivíduos/famílias. Ao considerarmos a adoção de um padrão alimentar saudável, o Guia Alimentar para a População Brasileira apresenta diretrizes importantes e direcionadas à prevenção de doenças para todos os estágios de vida. Em princípio, a alimentação saudável deve ser pautada por uma combinação de todos os grupos alimentares, com quantidade e qualidade adequadas (BRASIL, 2014). Além disso, um dos pilares da alimentação saudável compreende o consumo de 400 gramas de frutas e verduras diariamente, fator importante na adequação do consumo alimentar e para a prevenção de DCNTs (WORLD HEALTH ORGANIZATION, 2002).

No presente estudo, considerando somente a frequência semanal de consumo, 8,2\% e $13,1 \%$ das entrevistadas, respectivamente, mencionaram consumir frutas e saladas cruas diariamente. Essa informação torna-se ainda mais relevante ao considerarmos os efeitos de uma alimentação inadequada à própria saúde e também a influência no consumo alimentar familiar, uma vez que a maioria das mulheres afirmou ser responsável pelas escolhas alimentares da família.

Em vista de seu importante conteúdo nutricional, o baixo consumo de leite também desperta preocupação. Somente $14,8 \%$ da amostra apontou consumo diário, enquanto que $29,5 \%$ das entrevistadas mencionaram que nunca ou quase nunca consumiam leite. Sabe-se que o leite é importante fonte de cálcio e a ingestão adequada representa um fator modificável que pode atuar na prevenção de doenças musculoesqueléticas, como a osteoporose (KERSCHANSCHINDL, 2016).

Para as participantes desta pesquisa, com idade média de $30,1( \pm 6,7)$ anos, a ingestão recomendada de cálcio é de 1000mg/dia (INSTITUTE OF MEDICINE, 2011). Para suprir essa demanda, recomenda-se o consumo diário de dois a três copos de leite e/ou iogurte (BRASIL, 2008). Tendo em vista que este estudo voltou-se para a identificação da frequência de consumo, sem quantificar o número de porções ingeridas, esta última informação poderia contribuir para maior exatidão 
da quantidade em mg do nutriente em questão e, ao mesmo tempo, possivelmente demonstrar consumo de cálcio ainda mais insuficiente baseado na quantidade realmente consumida.

Sobre o consumo de feijão, tradicional na alimentação do brasileiro, constatou-se consumo diário por 50,8\% da amostra, e 24,6\% das participantes o consumiram com frequência de 5 a 6 dias por semana. O consumo regular de feijão (cinco ou mais dias na semana) é considerado um marcador de alimentação saudável (INSTITUTO BRASILEIRO DE GEOGRAFIA E ESTATÍSTICA, 2014b). Pereira et al. (2015), tendo como referência análise de dados de consumo de alimentos obtidos por meio da pesquisa VIGITEL, mostraram associação inversa entre o consumo regular de feijão e dislipidemias. Ou seja, os entrevistados que não consumiam feijão regularmente apresentavam prevalência de dislipidemia 32\% maior do que aqueles com o consumo adequado.

Considerando os hábitos alimentares que, quando mantidos rotineiramente e em quantidades excessivas, apresentam-se relacionados ao desenvolvimento de DCNTs, a presente pesquisa constatou que $60,7 \%$ e $54,1 \%$ das mulheres registraram ingestão de refrigerantes ou sucos artificiais e doces com frequência de três ou mais dias na semana. Esse resultado pode representar ingestão excessiva de açúcar simples, elemento que favorece o ganho de peso de adultos e crianças (WORLD HEALTH ORGANIZATION, 2015).

Alves, Muniz e Vieira (2013), fundamentados em dados da Pesquisa Nacional de Demografia e Saúde (PNDS), conduzida em 2006, mostraram que, para aquele ano, 82\% das crianças brasileiras, menores de cinco anos, já consumiam refrigerantes (considerado fonte de açúcar simples) pelo menos uma vez na semana, fato que pode contribuir para a perpetuação de um hábito alimentar inadequado na vida adulta. Os autores também relataram que o elevado consumo dessas bebidas está associado à diminuição da ingestão de leite e suco natural. Outro estudo realizado em 2015 mostrou que crianças brasileiras de 4 a 6 anos de idade consumiam açúcar além das recomendações diárias ( $5 \%$ acima da recomendação diária) e as crianças do Centro-Oeste chegaram a ultrapassar em 31,5\% esse limite, sendo o maior valor entre as regiões brasileiras. Ressalta-se que as crianças extrapolaram o limite apenas na soma dos lanches da manhã e da tarde, com destaque para o consumo de biscoitos recheados e bebidas açucaradas (FISBERG et al., 2015). A frequência elevada de consumo de doces, refrigerantes e/ou sucos artificiais pela mãe, poderia constituir fator de influência à família, uma vez que, conforme mencionado anteriormente, mais de $80 \%$ das entrevistadas consideravam-se responsáveis pelas escolhas alimentares/consumo de alimentos da família.

Um padrão alimentar pautado por preparações de alta densidade energética, rico em gordura e açúcar e escasso em frutas, verduras e legumes, pode contribuir para o desenvolvimento de DCNTs, como a obesidade (NEIA MARTINI; BORGES; GUEDES, 2014). Em nosso estudo, embora sem diferença significativa entre consumo alimentar e estado nutricional, foi possível verificar que o excesso de peso (sobrepeso e obesidade) foi a condição nutricional mais frequente $(50,8 \%)$. Além disso, proporção expressiva $(55,7 \%)$ de mulheres apresentaram valores de Circunferência da cintura superiores ao valor desejável, o que potencializa o risco de agravos cardiovasculares. Tais constatações podem refletir um padrão alimentar inadequado, evidenciado pela reduzida proporção de mulheres que consomem frutas, salada e leite diariamente e pelo elevado consumo semanal de bebidas açucaradas e doces. Sugere-se ainda que a maior frequência diária de consumo de salada por mulheres obesas, detectada nesta amostra, possa ser decorrente do maior consumo de alimentos de maneira geral, contudo, tal constatação requer investigação mais detalhada. 
O excesso de peso, principalmente a obesidade, tem sido associado ao desenvolvimento de doenças cardiovasculares, hipertensão arterial, diabetes mellitus tipo 2, dislipidemias, acidente vascular cerebral, câncer e doença renal crônica (WILLIAMS et al., 2015). Entre as mulheres, detectou-se associação também com problemas ginecológicos, alterações hormonais (WILLIAMS et al., 2015) e câncer de mama (segundo câncer mais prevalente em mulheres brasileiras), segundo dados do Instituto Nacional de Câncer (INCA) (2015).

No Brasil, o número de casos de indivíduos com excesso de peso tem sofrido aumentos substanciais. Considerando exclusivamente as mulheres de Campo Grande, MS, em 2006, primeira edição desta série de levantamentos, 36,7\% das campo-grandenses tinham excesso de peso e, 11,7\%, obesidade, sendo a décima segunda maior prevalência entre as capitais brasileiras (BRASIL, 2007). Em 2018, ano do último levantamento, as mulheres campo-grandenses ocupavam o sexto lugar no ranking de excesso de peso $(56,1 \%)$, considerando as 26 capitais brasileiras e o Distrito Federal. Para a obesidade, 21,5\% das mulheres apresentam tal condição (BRASIL, 2019).

Sabe-se que o estado nutricional pode sofrer influência da escolaridade (COSTA et al., 2014; MAGALHÃES et al., 2015). Segundo análise dos dados da VIGITEL, de 2018, especialmente entre as mulheres, a frequência de excesso de peso diminuiu, uniformemente, com o aumento do nível de escolaridade. Tanto para o excesso de peso quanto para a obesidade, as maiores prevalências concentraram-se no estrato de 0 a 8 anos de escolaridade, para ambos os sexos (BRASIL, 2019). No presente trabalho foi possível observar que 77\% das mulheres tinham 12 ou menos anos de estudo, contudo não foi constatada diferença significativa de acordo com o estado nutricional e anos de escolaridade.

A associação entre IA e a presença de obesidade foi demonstrada em mulheres mexicanas (Morales-Ruán et al., 2014). Os autores relataram que alimentos altamente calóricos frequentemente são menos onerosos, sendo acessíveis à população mais carente, consequentemente, facilitando o consumo. Morais et al. (2014), baseados em estudo de revisão, também observaram relação entre IA e obesidade em mulheres. Ainda enfatizaram que tanto casos de baixo peso, quanto de excesso de peso, podem estar presentes entre indivíduos com Segurança Alimentar, prevendo-se inadequações principalmente qualitativas no consumo alimentar. Contudo, em nosso estudo, não foi detectada diferença significativa entre IA e estado nutricional. Mesmo assim, as proporções de mulheres inseridas em domicílios em IA e com excesso de peso chamam a atenção, principalmente pelo risco de desenvolvimento de DCNTs associadas à alimentação.

Por fim, este estudo questionou a responsabilidade pelas escolhas alimentares da família, entendendo-as como a rotina de consumo alimentar. Acredita-se que as preferências alimentares das mães, pautadas por opções adequadas ou não e direcionadas de acordo com a renda e conhecimento nutricional, possam influenciar o padrão alimentar dos filhos. Nesse cenário, tais escolhas podem contribuir decisivamente para a instalação do excesso de peso e possíveis carências nutricionais entre todos os membros da família, notadamente entre as crianças, cujo hábito alimentar encontra-se em intensa fase de formação.

Em estudo transversal realizado com 357 famílias brasileiras, foi constatado que a mãe com acúmulo de gordura abdominal aumenta em 2,7 vezes a chance de a criança também apresentar a mesma condição. Esse desfecho, segundo os autores, pode associar-se tanto a fatores genéticos quanto socioculturais e de hábitos familiares (MELZER et al., 2015). Sabe-se também que a figura materna tende a induzir mais as crianças a comer do que a paterna, e as crianças com sobrepeso eram as mesmas com maior encorajamento para comer, cerca de 30 a 
36 encorajamentos durante uma refeição (RAMOS; STEIN, 2000). Esse encorajamento pode ser relacionado também aos alimentos de conteúdo nutricional inadequado.

A figura materna indubitavelmente assume representatividade em todo o contexto familiar, especialmente para a saúde e educação. Para que essa representatividade produza cada vez mais benefícios, é necessário que a mulher empodere-se de conhecimento, nesse caso especificamente no que diz respeito à alimentação, de forma a repassá-lo para seus filhos e futuras gerações. A Educação Alimentar e Nutricional, como estratégia de educação para a saúde, assume papel essencial na construção de hábitos alimentares saudáveis em todas as fases da vida, visando à Promoção da Saúde.

Cabe ainda ressaltar que, no Brasil, a Educação Alimentar e Nutricional vem integrando políticas públicas em diversos setores, fomentando a promoção da saúde a partir de mudanças de comportamentos alimentares e, consequentemente, a redução de riscos nutricionais. Contudo a atuação do nutricionista, pautada em fundamentação teórico-metodológica adequada a cada realidade e, notadamente nos serviços socioassistenciais, potencializaria o processo educativo e seus resultados (SIMON; RAMOS; ROSA, 2017).

\section{CONCLUSÃO}

A presente pesquisa identificou percentual de famílias com algum grau de IA superior ao constatado no último inquérito nacional com amostra do Estado de Mato Grosso do Sul, percentual esse possivelmente influenciado pelo cenário político-econômico do País, com redução do poder de compra dos brasileiros, especialmente com prejuízos na alimentação diária. Mesmo que a limitação da amostra represente um universo restrito, ao mesmo tempo, este estudo possibilitou detalhamento de variáveis relacionadas ao conceito global de Segurança Alimentar e Nutricional, a partir da análise do estado nutricional e do consumo de alimentos, representando um estudo exploratório inicial para a realidade local.

Os hábitos alimentares, evidenciados pela proporção de participantes que manifestaram consumo insuficiente, principalmente de frutas, verduras e leite, além daquelas que relataram consumo de refrigerantes e doces frequentemente, aponta para um padrão de consumo, que rotineiramente, poderá potencializar o risco para a instalação/manutenção do excesso de peso, já constatado em mais da metade das mulheres, e também de suas comorbidades. Sugere-se a implementação de estratégias de promoção à saúde, especialmente de Educação Alimentar e Nutricional, de âmbito municipal, voltadas para grupos em vulnerabilidade alimentar e nutricional. Ainda, a capacitação de gestores locais poderia contribuir para a identificação de tais situações e possibilitar o planejamento de ações imediatas e específicas para cada comunidade.

\section{REFERÊNCIAS}

ALVES, M. N.; MUNIZ, L. C.; VIEIRA, M. F. A. Consumo alimentar entre crianças brasileiras de dois a cinco anos de idade: Pesquisa Nacional de Demografia e Saúde (PNDS), 2006. Ciência \& Saúde Coletiva, Rio de Janeiro, v. 18, n. 11, p. 3369-77, nov. 2013.

ASSOCIAÇÃO BRASILEIRA DE EMPRESAS DE PESQUISA. Critério de Classificação Econômica Brasil. São Paulo: ABEP, 2014.

BRASIL. Ministério da Saúde. Secretaria de Vigilância em Saúde. Departamento de Vigilância de Doenças e Agravos não Transmissíveis e Promoção da Saúde. Vigitel Brasil 2018: vigilância de fatores de risco e 
proteção para doenças crônicas por inquérito telefônico - estimativas sobre frequência e distribuição sociodemográfica de fatores de risco e proteção para doenças crônicas nas capitais dos 26 estados brasileiros e no Distrito Federal em 2018. Brasília: Ministério da Saúde, 2019. 132 p.

BRASIL. Ministério da Saúde. Secretaria de Vigilância em Saúde. Departamento de Vigilância de Doenças e Agravos não Transmissíveis e Promoção da Saúde. Vigitel Brasil 2014: vigilância de fatores de risco e proteção para doenças crônicas por inquérito telefônico. Brasília: Ministério da Saúde, 2015. 152 p.

BRASIL. Ministério da Saúde. Secretaria de Atenção à Saúde. Departamento de Atenção Básica. Guia alimentar para a população brasileira. 2. ed. Brasília: Ministério da Saúde, 2014. 151 p.

BRASIL. Ministério da Saúde. Secretaria de Atenção à Saúde. Guia alimentar para a população brasileira: promovendo a alimentação saudável. Brasília: Ministério da Saúde, 2008. 210 p.

BRASIL. VIGITEL BRASIL 2006. Vigilância de Fatores de Risco e Proteção para Doenças Crônicas por Inquérito Telefônico. Estimativas sobre frequência e distribuição sócio-demográfica de fatores de risco e proteção para doenças crônicas nas capitais dos 26 estados brasileiros e no Distrito Federal em 2006. Brasília, DF: Ministério da Saúde, 2007. 92 p.

BRASIL. Presidência da República. Lei n. 11.346, de 15 de setembro de 2006. Cria o Sistema Nacional de Segurança Alimentar e Nutricional. Brasília, DF, 2006.

CABRAL, C. S.; LOPES, A. G.; LOPES, J. M.; VIANNA, R. P. T. Segurança alimentar, renda e Programa Bolsa Família: estudo de coorte em municípios do interior da Paraíba, Brasil, 2005-2011. Cadernos de Saúde Pública, Rio de Janeiro, v. 30, n. 2, p. 393-402, fev. 2014.

CÂMARA INTERMINISTERIAL DE SEGURANÇA ALIMENTAR E NUTRICIONAL. Plano Nacional de Segurança Alimentar e Nutricional (PLANSAN 2016-2019). Brasília: CAISAN; 2016.

COSTA, E. C.; LIRA, P. I. C. de; OLIVEIRA, J. S.; MENEZES, R. C. E. de; TAVARES, F. C. L. P.; BATISTA FILHO, M. Evolução do excesso de peso e fatores associados em mulheres de 10 a 49 anos em Pernambuco, Nordeste, Brasil. Revista de Nutrição, Campinas, v. 27, n. 5, p. 513-24, set./out. 2014.

FISBERG, M.; DEL'ARCO, A. P. W. T.; PREVIDELLI, A. N.; TOSATTI, A. M.; NOGUEIRA-DE-ALMEIDA, C. A. O (doce) lanche da criança brasileira: um retrato inédito. Fórum Saúde, 2015. Disponível em: http:// nutribrasilinfancia.com.br/mobile/pdf/Estudo_infografado.pdf. Acesso em: 2 jan. 2018.

INSTITUTE OF MEDICINE. Dietary Reference Intakes for Calcium and Vitamin D. Washington, D.C.: National Academy Press, nov. 2011.

INSTITUTO BRASILEIRO DE GEOGRAFIA E ESTATÍSTICA. Pesquisa Nacional por Amostra de Domicílios: segurança alimentar 2013. Rio de Janeiro: IBGE, 2014a.

INSTITUTO BRASILEIRO DE GEOGRAFIA E ESTATÍSTICA. Pesquisa Nacional de Saúde 2013: percepção do estado de saúde, estilos de vida e doenças crônicas. Rio de Janeiro: IBGE, 2014b.

INSTITUTO NACIONAL DE CÂNCER JOSÉ ALENCAR GOMES DA SILVA. Coordenação de prevenção e vigilância. Estimativa 2016: incidência de câncer no Brasil. Rio de Janeiro: INCA, 2015. 122 p.

KEPPLE, A. W.; SEGALL-CORRÊA, A. M. Conceituando e medindo segurança alimentar e nutricional. Ciência \& Saúde Coletiva, Rio de Janeiro, v. 16, n. 1, p. 187-99, jan. 2011.

KERSCHAN-SCHINDL, K. Prevention and rehabilitation of osteoporosis. Wiener Medizinische Wochenschrift, Austria, v. 166, n. 1-2, p. 22-7, fev. 2016. 
MAGALHÃES, T. C. A.; VIEIRA, S. A.; PRIORE, S. E.; RIBEIRO, A. Q.; FRANCESCHINI, S. C. C.; SANT'ANA, L. F. R. Fatores associados à dislipidemia em crianças de 4 a 7 anos de idade. Revista de Nutrição, Campinas, v. 28, n. 1, p. 17-28, jan./fev. 2015.

MAZUR, C. E.; NAVARRO, F. Insegurança alimentar e obesidade em adultos: Qual a relação? Saúde (Santa Maria), Santa Maria, v. 41, n. 2, p. 35-44, jul./dez. 2015.

MELZER, M. R. T. F.; MAGRINI, I. M.; DOMENE, S. M. A.; MARTINS, P. A. Fatores associados ao acúmulo de gordura abdominal em crianças. Revista Paulista de Pediatria, São Paulo, v. 33, n. 4, p. 437-44, out./dez. 2015.

MORAIS, D. C.; DUTRA, L. V.; FRANCESCHINI, S. C. C.; PRIORE, S. E. Insegurança Alimentar e indicadores antropométricos, dietéticos e sociais em estudos brasileiros: uma revisão sistemática. Ciência \& Saúde Coletiva, Rio de Janeiro, v. 19, n. 5, p. 1475-88, jan./maio 2014.

MORALES-RUÁN, M. C.; HUMARÁN, I. M. G.; SHAMAH-LEVY, T.; VALDEMARRA-ÁLVAREZ, Z.; MELGARQUIÑONEZ, H. La Inseguridad alimentaria está asociada com obesidad em mujeres adultas de México. Salud Pública de México, Méxic], v. 56, Supl. 1, p. 54-61, fev. 2014.

NEIA MARTINI, F. A.; BORGES, M. B.; GUEDES, D. P. Hábito alimentar e síndrome metabólica em uma amostra de adultos brasileiros. Archivos Latinoamericanos de Nutrición, Venezuela, v. 64, n. 3, p. 161-73, set. 2014.

PEREIRA, L. P.; SICHIERI, R.; SEGRI, N. J.; SILVA, R. M. V. G. da; FERREIRA, M. G. Dislipidemia autorreferida na região Centro-Oeste do Brasil: prevalência e fatores associados. Ciência \& Saúde Coletiva, Rio de Janeiro, v. 20, n. 6, p. 1815-24, jun. 2015.

RAMOS, M.; STEIN, L. M. Desenvolvimento do comportamento alimentar infantil. Jornal de Pediatria, São Paulo, v. 76, supl. 3, p. 229-37, 2000.

SEGALL-CORRÊA, A. M.; ESCAMILLA, R. P.; SAMPAIO, M. F. A.; MARIN-LEON, L.; PANIGASSI, G.; MARANHA, L. K.; BERGAMASCO, S.; OLIVEIRA, J. Relatório Técnico. Acompanhamento e avaliação da segurança alimentar de famílias brasileiras: validação de metodologia e de instrumento de coleta de informação. Campinas: UNICAMP, 2004.

SEGALL-CORRÊA, A. M.; MARIN-LEON, L.; HELITO, H.; PÉREZ-ESCAMILLA, R.; SANTOS, L. M. P.; PAES-SOUSA, R. Transferência de renda e segurança alimentar no Brasil: análise dos dados nacionais. Revista de Nutrição, Campinas, v. 21, suplemento, p. 39-51, jul./ago. 2008.

SIMON, L.; RAMOS, M.; ROSA, R. S. Análise dos Cadernos sobre Educação Alimentar e Nutricional voltados aos serviços socioassistenciais. Demetra, Rio de Janeiro, v. 12, n. 4, p. 1033-49, 2017.

VOCI, S. M.; SLATER, B. Consistência interna da Escala Brasileira de Insegurança Alimentar aplicada por entrevista telefônica e preenchida pelo entrevistado. Nutrire, São Paulo, v. 40, n. 3, p. 318-27, dez. 2015.

WILLIAMS, E. P.; MESIDOR, M.; WINTERS, K.; DUBBERT, P. M.; WYATT, S. B. Overweight and obesity: prevalence, consequences, and causes of a growing public health problem. Current Obesity Reports, United States, v. 4, n. 3, p. 363-70, set. 2015.

WORLD HEALTH ORGANIZATION. OMS recomenda cortar pela metade o consumo de açúcar, 2015. Disponível em: http://nacoesunidas.org/com-novas-evidencias-oms-corta-pela-metade-consumo-idealde-acucar/. Acesso em: 15 jan. 2018.

WORLD HEALTH ORGANIZATION. Waist circunference and waist-hip ratio: report of a who expert consultation. Geneva, 2008. 
WORLD HEALTH ORGANIZATION. Global strategy on diet, physical activity and health. 2002. Disponivel em: http://www.who.int/dietphysicalactivity/publications/releases/pr84/en/. Acesso em: 10 jan. 2018.

WORLD HEALTH ORGANIZATION. Physical status: the use and interpretation of anthropometry. Who Technical Report Series 894. Geneva, 1995.

\section{Sobre os autores:}

Lídia Viegas Tenório da Silva Santos - Especialização em Paciente Crítico, Urgência e Emergência pela Residência Multiprofissional em Saúde - Atenção ao Paciente Crítico, da Universidade Federal Mato Grosso do Sul (UFMS). Graduada em Nutrição pela UFMS. E-mail: lidia.viegass@gmail.com, Orcid: http://orcid.org/0000-0003-1223-2311

Larissa de Azevedo Cáceres - Especialização em Paciente Crítico, Urgência e Emergência pela Residência Multiprofissional em Saúde - Atenção ao Paciente Crítico, da Universidade Federal Mato Grosso do Sul (UFMS). Graduada em Nutrição pela UFMS. E-mail: larissacaceres@outlook.com, Orcid: http://orcid.org/0000-0002-2302-5433

Giovana Eliza Pegolo - Doutora em Alimentos e Nutrição pela Universidade Estadual Paulista (UNESP) e Mestre em Ciências pela Universidade de São Paulo (USP). Docente do Curso de Nutrição e do Programa de Residência Multiprofissional em Atenção ao Paciente Crítico, da Universidade Federal de Mato Grosso do Sul (UFMS). E-mail: giovana.pegolo@ufms.br, Orcid: http://orcid.org/0000-0003-2636-2547 